\title{
Basin of Attraction Determines Hysteresis in Explosive Synchronization
}

\author{
Yong Zou, ${ }^{1,2}$ Tiago Pereira, ${ }^{3,4}$ Michael Small, ${ }^{5}$ Zonghua Liu, ${ }^{1}$ and Jürgen Kurths ${ }^{2,6}$ \\ ${ }^{1}$ Department of Physics, East China Normal University, Shanghai 200062, China \\ ${ }^{2}$ Potsdam Institute for Climate Impact Research, P.O. Box 601203, 14412 Potsdam, Germany \\ ${ }^{3}$ Department of Mathematics, Imperial College London, London SW72AZ, United Kingdom \\ ${ }^{4}$ London Mathematical Laboratory, London WC2N 6DF, United Kingdom \\ ${ }^{5}$ School of Mathematics and Statistics, University of Western Australia, Crawley, Western Australia 6009, Australia \\ ${ }^{6}$ Institute for Complex Systems and Mathematical Biology, University of Aberdeen, Aberdeen AB243UE, United Kingdom
}

(Received 8 January 2014; revised manuscript received 11 February 2014; published 18 March 2014)

\begin{abstract}
Spontaneous explosive emergent behavior takes place in heterogeneous networks when the frequencies of the nodes are positively correlated to the node degree. A central feature of such explosive transitions is a hysteretic behavior at the transition to synchronization. We unravel the underlying mechanisms and show that the dynamical origin of the hysteresis is a change of basin of attraction of the synchronization state. Our findings hold for heterogeneous networks with star graph motifs such as scale-free networks, and hence, reveal how microscopic network parameters such as node degree and frequency affect the global network properties and can be used for network design and control.
\end{abstract}

DOI: 10.1103/PhysRevLett.112.114102

PACS numbers: 05.45.Xt, 05.45.Ac, 89.75.Hc

Emerging abrupt transitions are ubiquitous in complex systems and play a crucial role in human society and a wide variety of fields [1]. In particular, abrupt transitions to synchronization in networks with heterogenous degree distribution have attracted much attention. Previous works suggest that such transitions are due to a positive correlation between the frequency and degree of the node [2-9]. Abrupt transition has been observed in scale-free (SF) networks [2], electronic circuits [10], time delayed systems [11], and a second order Kuramoto model [12].

A central feature of these emerging abrupt transitions is a hysteretic behavior at the onset of synchronization. As the interaction strength is increased adiabatically, the network experiences a fast explosive jump from an incoherent state to a coherent one. Moreover, there is a sudden drop from the coherent state to the incoherent one when the coupling strength is progressively decreased in the backward direction. These two curves (called forward and backward continuation below, respectively) do not overlap, instead, showing a hysteretic behavior. The hysteresis in abrupt transitions is due to the network interaction and, hence, opens new paradigms for network control as coherence and incoherence coexist in the hysteresis loop. Despite this great interest, hysteresis at the transition to synchronization remains elusive. In particular, it is unclear on a microscopic level how network parameters affect the critical coupling thresholds (the hysteresis loop) and what the dynamical origins are for the hysteresis associated with explosive synchronization.

In this Letter, we investigate hysteresis associated with the explosive transition scenario first in networks with a star graph motif, and then in generic SF networks. Our results reveal that correlation in frequency degree leads to the existence of a phase locking state and that the hysteretic behavior is attributed to the basin of attraction of phase locking. The phase locking state ceases to exist at a critical parameter $\lambda_{c}^{b}$, corresponding to synchronization loss coming from a coherent state. Starting from an incoherent state and moving toward coherence, our analysis suggests that the locking manifold changes its basin of attraction at a critical parameter $\lambda_{c}^{f}$ and the locking manifold becomes globally attractive. We find that, whereas the backward coupling threshold $\lambda_{c}^{b}$ tends to a constant value for large networks, the forward critical $\lambda_{c}^{f}$ scales with the system size.

In a heterogeneous network such as SF networks, hubs play a dominant role for both structural organization [13] and dynamical processes [14], e.g., providing substantial resilience for preventing cascading failures [15-17]. Hubs are modeled as star motifs. A star is composed of $K(K \geq 2)$ peripheral nodes (or leaves) connected to the hub. Let us start by keeping the same setting for the frequency-degree correlation as initially explained in [2]. The hub has a frequency $\omega_{K+1}=K \omega$, while all the leaves have the same frequency $\omega_{j}=\omega$ for $1 \leq j \leq K$. Later on, we will generalize to nonidentical leaf nodes. The equations of motion are

$$
\begin{gathered}
\dot{\phi}_{K+1}=K \omega+\lambda \sum_{j=1}^{K} \sin \left(\phi_{j}-\phi_{K+1}\right), \\
\dot{\phi}_{j}=\omega+\lambda \sin \left(\phi_{K+1}-\phi_{j}\right), \quad \text { for } 1 \leq j \leq K,
\end{gathered}
$$

where $\phi_{K+1, j}$ are phase dynamics of the hub and leaf nodes, respectively, $\lambda$ is the coupling strength. The Kuramoto order parameter $\mathcal{R}(t)$ is defined as $\mathcal{R}(t) e^{i \Psi(t)}=$ $\sum_{j=1}^{K+1} e^{i \phi_{j}} /(K+1)$. We quantify coherence by $r=\langle\mathcal{R}(t)\rangle_{T}$, 
where $\langle\cdot\rangle_{T}$ denotes a time average with $T \gg 1$. Small values of the parameter $r$ indicate incoherent behavior. In contrast, as $r \rightarrow 1$ we encounter a highly coherent state.

Main results. - The backward critical coupling $\lambda_{c}^{b}$ and the forward critical coupling $\lambda_{c}^{f}$ are determined by local and global attractivity properties of a locking manifold $M_{a}$, respectively. Hence, the basin of attraction of $M_{a}$ governs the onset of hysteresis. Moreover, the scaling relationships of the coupling thresholds on the degree $K$ are given by $\lambda_{c}^{b} \rightarrow \omega$, and $\lambda_{c}^{f} \propto K^{1 / 2} \omega$, for $K \gg 1$, respectively. Our results are based on the theory of invariant manifolds, and the recent new findings about persistence of synchronization $[18,19]$, together with the attractivity and basin of attraction [20].

Backward continuation-from coherence to incoherence.We start from a coherent state where the nodes are phase locked and decrease the coupling until we obtain a loss of coherence. We perform a local stability analysis to explain this scenario. The state space of Eqs. (1) and (2) is the $K+1$ dimensional torus $\mathbb{\pi}^{K+1}$. Consider $\Phi=\left(\phi_{1}, \ldots, \phi_{K+1}\right)$, and $\Omega_{k}=(\omega, \ldots, \omega, K \omega)$. Moreover, consider $\boldsymbol{H}: \mathbb{T}^{K+1} \rightarrow \mathbb{T}^{K+1}$ defined by $\boldsymbol{H}(\Phi)=$ $\left(\sin \left(\phi_{K+1}-\phi_{1}\right), \sin \left(\phi_{K+1}-\phi_{2}\right), \ldots, \sin \left(\phi_{K+1}-\phi_{K}\right)\right.$, $\left.\sum_{j=1}^{K} \sin \left(\phi_{j}-\phi_{K+1}\right)\right)$. With this notation, the equations of motion Eqs. (1) and (2) are rewritten in the compact form $\dot{\Phi}=\Omega_{k}+\lambda \boldsymbol{H}(\Phi)$. The locking manifold is defined by

$M_{a}:=\left\{\Phi \in \mathbb{T}^{K+1}: \phi_{1}=\cdots=\phi_{K} \quad\right.$ and $\left.\quad \phi_{K+1}-\phi_{1}=a\right\}$.

Notice that the nonzero value of $a$ determines the locking between the hub and the leaves. We show the existence conditions for $M_{a}$.

Solution curves in $M_{a}$ read as $\dot{\Phi}=\Omega_{k}-\lambda \boldsymbol{H}(\boldsymbol{a})$, with $\boldsymbol{a}=c(1, \ldots, 1)+(0, \ldots, 0, a)$, where $c$ is a real number. The solutions are $\Phi(t)=\left[\Omega_{k}-\lambda \boldsymbol{H}(\boldsymbol{a})\right] t+\Phi_{\mathbf{0}}$, where $\Phi_{0} \in M_{a}$, and satisfy the condition $\phi_{K+1}-\phi_{1}=a$, which yields the equation

$$
-(K-1) \omega+\lambda(K+1) \sin a=0 .
$$

Since $\omega, K$, and $\lambda$ are positive, a solution exists if $[(K-1) \omega] /[(K+1) \lambda] \leq 1$, which further leads to $0<a \leq$ $\pi / 2$ [21]. The equality determines the critical coupling strength for the existence of the locking manifold, which yields the critical coupling for the backward continuation curve as

$$
\lambda_{c}^{b}=\frac{(K-1) \omega}{K+1} .
$$

It turns out that whenever $M_{a}$ exists, it is locally attractive. To see this, we study the tangent dynamics to $M_{a}$. Consider $\Phi=\boldsymbol{\Psi}+\boldsymbol{\xi}$, where $\boldsymbol{\Psi}$ is a solution curve in $M_{a}$. The equation associated with $\boldsymbol{\xi}$ reads as $\dot{\xi}=-\lambda \cos a L_{s} \boldsymbol{\xi}+\boldsymbol{R}(\boldsymbol{\xi})$, where $L_{s}$ is the Laplacian matrix of the star graph, and $\boldsymbol{R}$ is a nonlinear term satisfying $\boldsymbol{R}(\boldsymbol{\xi}) \leq A\|\boldsymbol{\xi}\|$, for some constant $A$. The solution of the linear part can be represented as $\boldsymbol{\xi}(t)=\exp \left\{\lambda \cos a L_{s}(t-\tau)\right\} \boldsymbol{\xi}(\tau)$. Notice that $\xi \notin \operatorname{span}(1, \ldots, 1)$, otherwise, it could be absorbed in $\boldsymbol{\Psi}$. As the Laplacian is positive semi-definite with smallest nonzero eigenvalue equal to 1 , we get $\|\boldsymbol{\xi}(t)\| \leq C \exp \{-\lambda \cos a(t-\tau)\}\|\boldsymbol{\xi}(\tau)\|$ for some constant $C>1$. This implies that, whenever the manifold $M_{a}$ exists, it is locally stable. Moreover, since the bound is uniform $\tau$ and exponential, the stability will persist under the nonlinearities [19].

If $\lambda>\lambda_{c}^{b}$ and initial conditions are given close to the locking manifold $M_{a}$, the local attractivity of $M_{a}$ allows us to compute the order parameter explicitly, which reads

$$
r^{2}=\frac{K^{2}+1}{(K+1)^{2}}+\frac{2 K}{(K+1)^{2}} \sqrt{1-\left(\frac{(K-1) \omega}{(K+1) \lambda}\right)^{2}} .
$$

Now, note that as the locking manifold ceases to exist at $\lambda=\lambda_{c}^{b}$, the order parameter $r$ assumes a critical value

$$
r_{c}^{b}=\frac{\sqrt{K^{2}+1}}{K+1} .
$$

The above analysis explicitly determines the behavior of $r$ in the backward direction. The loss of coherence occurs at the point $\left(\lambda_{c}^{b}, r_{c}^{b}\right)$ [22].

Figure 1(a) shows the order parameter $r$ when the coupling is decreased in the backward direction and starting from a coherent state. The numerical results for various network sizes show precise agreement with the theoretical curve given by Eq. (5). We obtain the critical points $\left(\lambda_{c}^{b}, r_{c}^{b}\right)$ as predicted [denoted by coordinates in Fig. 1(a)]. In the regime of $\lambda<\lambda_{c}^{b}$, the $K$ star network is reduced to two groups: the hub and the set of leaves, evolving asynchronously. (a)

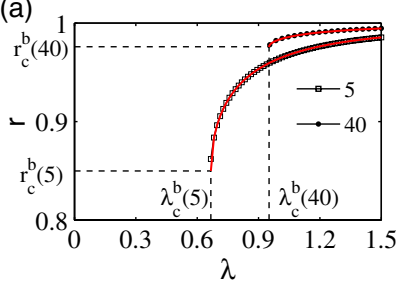

(b)

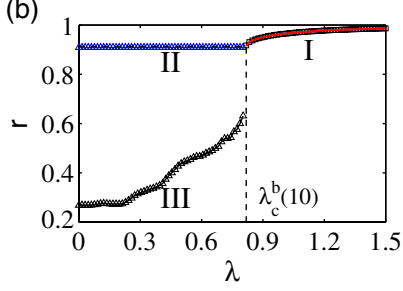

FIG. 1 (color online). Order parameter $r$ as a function of the coupling $\lambda$ for various sizes. The thick lines are theoretical curves obtained by Eq. (5). (a) $\left(K_{1}=5, K_{2}=40\right)$, and (b) comparison between with and without noise $(K=10) . r$ is an arithmetic mean value of $\left[\mathcal{R}_{\min }(t), \mathcal{R}_{\max }(t)\right]$ over 100 random realizations. Part II without noise while, for parts I and III, there is a random frequency mismatch $\omega_{j}=\omega+\zeta_{j}$ where $\zeta_{j} \in[-0.05,0.05]$ for leaf nodes. 
In the next case, we consider frequency mismatches for leaves

$$
\phi_{j}^{\prime}=\omega+\zeta_{j}+\lambda \sin \left(\phi_{K+1}-\phi_{j}\right), \quad \text { for } 1 \leq j \leq K,
$$

where $\zeta_{j}$ is a random variable uniformly distributed in $[-\varepsilon, \varepsilon]$. Notice that if $\varepsilon$ is small and the locking manifold is exponentially and uniformly attractive, these perturbations do not destroy the locking manifold [part I in Fig. 1(b)]. There exists another stable locking manifold in the neighborhood of $M_{a}$ for $\lambda>\lambda_{c}^{b}$. When $\lambda<\lambda_{c}^{b}$, the locking manifold no longer exists, and, as the leaves rotate at distinct frequencies, a drop in the order parameter is observed [part III in Fig. 1(b)]. In comparison, when no noise is introduced in Eq. (7), we find the absence of the sudden drop in $r$ which takes place as all leaves are identical [shown by part II in Fig. 1(b)].

Forward continuation-from incoherence to coherence.Starting from an incoherent state ( $r$ close to zero) and increasing the coupling strength leads to a transition towards coherence at a coupling threshold $\lambda_{c}^{f}>\lambda_{c}^{b}$. Our numerical investigations reveal that this behavior is related to the basin of attraction of the locking manifold $M_{a}$. At the first stage the locking manifold $M_{a}$ is only locally attractive, i.e., for $\lambda \in\left(\lambda_{c}^{b}, \lambda_{c}^{f}\right)$. Then, for $\lambda>\lambda_{c}^{f}$, the locking manifold is globally attractive, which means that, starting from an incoherent state for $\lambda>\lambda_{c}^{f}$, the network dynamics are attracted to $M_{a}$. In other words, for $\lambda>\lambda_{c}^{f}$, the phase difference is $\phi_{K+1}-\phi_{j}=a$, where $a=a(\lambda)$ given by Eq. (3). We reveal that this is, indeed, the case, as shown in Fig. 2(a) for distinct network sizes.

To analyze the basin of attraction, we draw initial conditions randomly from an interval $[-\delta, \delta]$ with $\delta \leq \pi$. Hence, if $\delta$ is close to $\pi$, the oscillators start from an incoherent state; in contrast, if $\delta$ is close to zero, all oscillators start at a coherent state. Hence, the value of $\delta$ enables us to capture the basin of attraction of $M_{a}$. For each pair $(\delta, \lambda)$, we compute the order parameter $r$, and the result is shown in Fig. 3. Note that, for $\lambda<\lambda_{c}^{b}$ and small values of
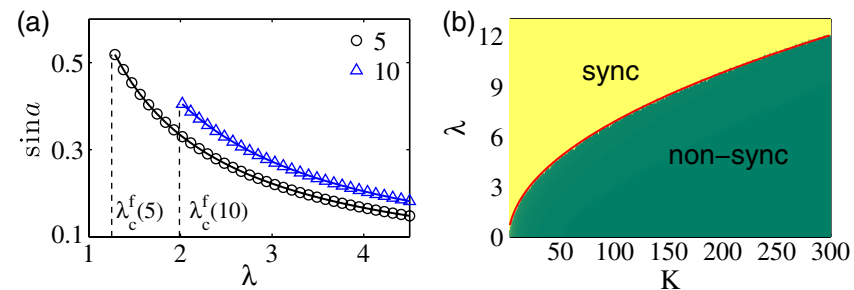

FIG. 2 (color online). (a) Phase difference $\sin a=\sin \left(\phi_{K+1}-\phi_{1}\right)$ between the hub and the first leaf as a function of $\lambda$ for $\lambda>\lambda_{c}^{f}$. Both circles and triangles represent the numerical simulation, and the thick lines are theoretical prediction provided by Eq. (3). Network size: $K=5(\circ), K=10(\Delta)$. (b) Order parameter $r$ on the parameter space of $(K, \lambda)$ for the forward continuation. The (red) thick line is from fitting the scaling relation provided by Eq. (8), where the parameter $1 / B=0.6989$. $\delta$ (e.g., $\delta=0$ ), the order parameter is close to one (shown by the bright area in Fig. 3), as all leaves are synchronized forming a group against the hub. In this regime, $r \approx$ $(\sin \delta) / \delta$ [23], explaining why high values of $r$ are observed for small $\delta$.

For $\lambda>\lambda_{c}^{f}$ and $\delta=\pi$, the oscillators start at an incoherent state and then tend to the locking manifold $M_{a}$ leading $r$ to be close to 1 . This scenario is not affected by the presence of mismatches in the oscillator frequencies as shown by Fig. 3(b).

While the value $\lambda_{c}^{b}$ tends to a constant, the critical forward coupling $\lambda_{c}^{f}$ scales roughly as $K^{1 / 2}$. Hence, for large $K$, the difference between the forward and backward coupling thresholds becomes severe. Note that our results below correspond to $\delta=\pi$, namely, the initial conditions are randomly chosen from $[-\pi, \pi]$. We calculate the order parameter $r$ for various network sizes, $K \in[3,300]$ for each coupling strength $\lambda$, yielding a color coded parameter space of $(K, \lambda)$ as shown in Fig. 2(b), where we observe an abrupt transition from an incoherent to a coherent state.

To obtain an analytical understanding of this scaling property for $\lambda_{c}^{f}$, we use the theory recently developed in Ref. [18]. To this end, we write this phase locking problem between the hub and leaves as a perturbation of an identical synchronization problem. Hence, the isolated dynamics of the hub reads as $\dot{\phi}_{K+1}=\omega+g_{K+1}$. Representing Eqs. (1) and (2) in block form yields the perturbation $\boldsymbol{G}=(0, \ldots, 0,(K-1) \omega)$. The block equation then reads $\dot{\Phi}=\Omega+\boldsymbol{H}(\Phi)+\boldsymbol{G}$, where $\Omega=(\omega, \cdots, \omega)$. After an involved algebraic manipulation following Refs. [18,19], we obtain $\left(\sum_{j}\left|\phi_{K+1}-\phi_{j}\right|^{2}\right)^{1 / 2} \leq(\sigma\|G\|) / \lambda$, where $\|\cdot\|$ denotes the Euclidean norm, and $\sigma$ is a constant.

Hence, starting from an incoherent state to obtain a coherent one, if the trajectories enter the neighborhood of a fully synchronized state, $\phi_{1}=\cdots=\phi_{K+1}$. This neighborhood contains the locking manifold $M_{a}$ as $a$ tends to zero. Using the above bounds for the phase difference, we obtain the scaling behavior of the coupling parameter. Indeed,
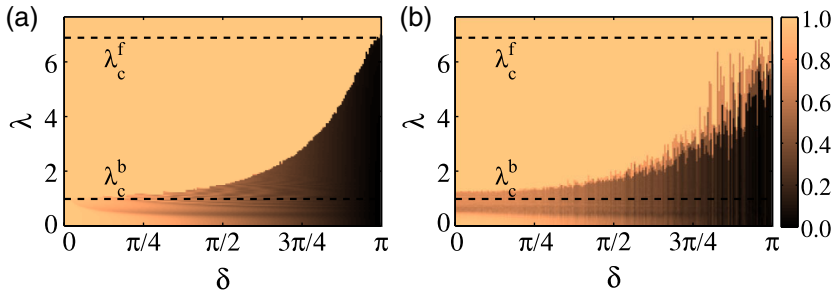

FIG. 3 (color online). Order parameter $r$ on the parameter space $(\delta, \lambda)$ for the forward continuation $(K=100)$. Initial conditions are randomly drawn from an interval $[-\delta, \delta]$. The horizontal dashed lines are critical couplings $\lambda_{c}^{b}$ and $\lambda_{c}^{f}$ from the theory. (a) without noise effect, (b) with frequency mismatches for leaf nodes, namely, $\omega_{j}=\omega+\zeta_{j}$ where the random value $\zeta_{j} \in[-0.01,0.01]$. 
notice that $\|\boldsymbol{G}\|=(K-1) \omega$, and using that the oscillators start from a incoherent state $\left|\phi_{K+1}-\phi_{j}\right|<2 \pi$, we obtain $\left(\sum_{j}\left|\phi_{K+1}-\phi_{j}\right|^{2}\right)^{1 / 2} \leq 2 \pi \sqrt{K}$. Manipulating this equation, we obtain that the coupling strength scales $\lambda \propto[(K-1) \omega] / \sqrt{K}$. This coupling corresponds to the necessary condition to get coherence starting from an incoherent state. However, this is precisely the forward coupling strength $\lambda_{c}^{f}$. Hence, trajectories will approach the locking manifold with the coupling strength

$$
\lambda_{c}^{f} \approx\left(\frac{K-1}{\sqrt{K}} \frac{1}{B}\right) \omega
$$

where $B$ is a constant parameter [24]. Our numerical result in Fig. 2(b) shows an excellent agreement with this theoretical analysis.

Scale-free networks.-The results above for star graphs can be straightforwardly applied to explain the recent findings of hysteresis in SF networks when the mean degree is small, due to the role of hubs. If the average degree of the network is small, then the network can be seen as a collection of star graphs. In particular, this can be seen to be the case for random power-law graphs as the exponent $\gamma \rightarrow 3$ [13]. More generally, the role of hubs in scale-free networks is certainly dominant, and it is only for low values of $\gamma$ (i.e., $\gamma<2.5$ ) that one will expect graphs with more links than a tree and, hence, exhibiting an excess of loops and significant deviation from a composition of hubs [13]. Of course, experimentally observed SF networks are very rarely trees-nonetheless, they remain defined by their high degree hubs.

In such networks, each hub and its corresponding neighboring nodes of low degrees will have a locking manifold, and the connections between low degree nodes of distinct hubs act as small perturbations. Therefore, the investigation of the hysteresis-like behavior on a SF network can be greatly explained by a star graph with frequency mismatches for leaf nodes [i.e., Eq. (7)]. In combination with the global order parameter $r$, it is convenient to compute the local order parameter $r_{i}$ for the $i$ th hub. Parameter $r_{i}$ is obtained by averaging only over nodes connected to the $i$ th hub. The local order parameters play a role in the SF network as hubs are connected to a different number of nodes $K_{i}$.

We generate a SF network by means of the BarabásiAlbert model with $m_{0}=1[25,26]$. We analyze the hubs by the local order parameter $r_{i}$. As predicted by Eq. (4), the backward continuation for various hubs of different sizes converges to the critical value $\lambda_{c}^{b} \rightarrow \omega, K \gg 1$ [size independent shown by the backward curves of the two largest hubs in Fig. 4(a)]. In contrast, since hubs often do not have the same degrees, the local order parameter $r_{i}$ will present forward transitions at distinct coupling values but are still governed by Eq. (8). In Fig. 4(a), we show the forward curves for the two largest hubs of a network
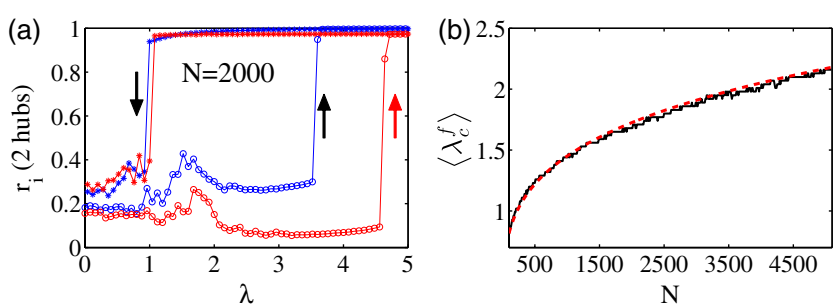

FIG. 4 (color online). (a) Local order parameter $r_{i}$ versus coupling strength for two chosen hubs $\left(K_{1}=39\right.$ red; $K_{2}=24$ blue; $\left.\lambda_{c, 1}^{f}=4.64, \lambda_{c, 2}^{f}=3.63\right)$. (b) Critical coupling $\left\langle\lambda_{c}^{f}\right\rangle$ vs sizes of SF networks, where $\langle\cdot\rangle$ is an ensemble average over 50 network realizations. The dashed line is the theoretical curve predicted by Eq. (9).

with 2000 nodes with degrees $K_{1}=39$ and $K_{2}=24$. Denote $\lambda_{c, 1}^{f}$ for the critical value of the largest hub, and $\lambda_{c, 2}^{f}$ for the second largest. Our results predict that $\lambda_{c, 1}^{f} / \lambda_{c, 2}^{f}=\sqrt{K_{1} / K_{2}}=1.275$, which is in an excellent agreement with our simulations yielding $\lambda_{c, 1}^{f} / \lambda_{c, 2}^{f}=$ 1.278. This result on the dominant role of hubs holds for networks of various sizes and random realizations.

We calculate the forward critical coupling $\lambda_{c}^{f}$ for various network sizes. For one network of size $N$, we numerically estimate the threshold $\lambda_{c}^{f}$ by fixing a level of coherence over hubs (say $r=0.5$ over the top 20 hubs). In addition, we consider the expectation of $\left\langle\lambda_{c}^{f}\right\rangle$ with respect to the network ensemble. The dependence of the expected coupling $\left\langle\lambda_{c}^{f}\right\rangle$ on the system size follows: Note that the expected degree of the largest hub $K_{\max }$ scales as $N^{1 /(\gamma-1)}$ [27], which means that on average the hubs are star motifs with $N^{1 /(\gamma-1)}$ leaves. Our previous considerations show that

$$
\left\langle\lambda_{c}^{f}\right\rangle \propto N^{\frac{1}{2(\gamma-1)}} .
$$

This is in agreement with our numerical experiments on the SF network where $\gamma=3$, as shown in Fig. 4(b).

Nonetheless, if the mean degree is high enough (for large $m_{0}$ ), the leaves of the hubs will strongly interact. So the modeling of a SF network as a collection of stars is no longer useful. In such situations, mean field approaches may capture the behavior of the leaves [2,11]. An interesting question is when does the crossover between our approach and the mean field scheme take place. Judd [14] provides strong indication that, even when the approximation is not precise, modeling of a SF network as a collection of stars may still be useful-and indicates that SF networks that are collections of stars are actually quite common [13].

In summary, we have shown that the abrupt transition in the Kuramoto model in both star motifs and SF networks is associated with a locking manifold and its local and global attractivity properties. The critical coupling associated with loss of coherence is determined by the existence of a locking manifold, whereas the critical coupling responsible 
for attaining coherence starting from an incoherent state is related to a change in the basin of attraction of the locking manifold. We have uncovered the distinct dependence of both coupling thresholds on the network size, revealing that the hysteresis is stronger in large networks. Our findings provide methods for controlling the transition and hysteresis in terms of microscopic network parameters.

This work was partially supported by the National Natural Scientific Foundation of China grants (No. 11305062, No. 11135001), Specialized Research Fund for the Doctoral Program (No. 20130076120003) (Y.Z., Z.L.), Australian Research Council Future Fellowship No. FT110100896 (M.S.), Marie Curie IIF Fellowship (No. 303180), CNPq, and Leverhulme Trust Grant (No. RPG-279) (T.P.), and by the Government of the Russian Federation (Agreement No. 14.Z50.31.0033) (J. K.).

[1] S. N. Dorogovtsev, A. V. Goltsev, and J. F. F. Mendes, Rev. Mod. Phys. 80, 1275 (2008).

[2] J. Gómez-Gardeñes, S. Gómez, A. Arenas, and Y. Moreno, Phys. Rev. Lett. 106, 128701 (2011).

[3] T. Kaue Dal'Maso Peron and F. A. Rodrigues, Phys. Rev. E 86, 056108 (2012).

[4] P. S. Skardal, J. Sun, D. Taylor, and J. G. Restrepo, Europhys. Lett. 101, 20001 (2013).

[5] W. Liu, Y. Wu, J. Xiao, and M. Zhan, Europhys. Lett. 101, 38002 (2013).

[6] B. C. Coutinho, A. V. Goltsev, S. N. Dorogovtsev, and J. F. F. Mendes, Phys. Rev. E 87, 032106 (2013).

[7] P. Li, K. Zhang, X. Xu, J. Zhang, and M. Small, Phys. Rev. E 87, 042803 (2013).

[8] I. Leyva, A. Navas, I. Sendiña Nadal, J. A. Almendral, J. M. Buldú, M. Zanin, D. Papo, and S. Boccaletti, Sci. Rep. 3, 1281 (2013).

[9] X. Zhang, X. Hu, J. Kurths, and Z. Liu, Phys. Rev. E 88, 010802 (2013).

[10] I. Leyva, R. Sevilla-Escoboza, J. M. Buldú, I. Sendiña Nadal, J. Gómez-Gardeñes, A. Arenas, Y. Moreno,
S. Gómez, R. Jaimes-Reátegui, and S. Boccaletti, Phys. Rev. Lett. 108, 168702 (2012).

[11] T. Kaue Dal'Maso Peron and F. A. Rodrigues, Phys. Rev. E 86, 016102 (2012).

[12] P. Ji, T. Kaue Dal'Maso Peron, P. J. Menck, F. A. Rodrigues, and J. Kurths, Phys. Rev. Lett. 110, 218701 (2013).

[13] K. Judd, M. Small, and T. Stemler, Europhys. Lett. 103, 58004 (2013).

[14] K. Judd, Chaos 23, 043112 (2013).

[15] L. K. Gallos, R. Cohen, P. Argyrakis, A. Bunde, and S. Havlin, Phys. Rev. Lett. 94, 188701 (2005).

[16] T. Nishikawa, A.E. Motter, Y.-C. Lai, and F. C. Hoppensteadt, Phys. Rev. Lett. 91, 014101 (2003).

[17] T. Pereira, Phys. Rev. E 82, 036201 (2010).

[18] T. Pereira, D. Eroglu, G. B. Bagci, U. Tirnakli, and H. J. Jensen, Phys. Rev. Lett. 110, 234103 (2013).

[19] T. Pereira, J. Eldering, M. Rasmussen, and A. Veneziani, Nonlinearity 27, 501, (2014).

[20] P. J. Menck, J. Heitzig, N. Marwan, and J. Kurths, Nat. Phys. 9, 89 (2013).

[21] The fixed point of Eq. (3) in $(0, \pi / 2]$ is stable, while the other unstable fixed point in $(\pi / 2, \pi)$ is neglected.

[22] In the sense that, after this point, the order parameter $\mathcal{R}$ oscillates.

[23] The initial conditions $\phi_{j}^{0} \mathrm{~s}$ are drawn independently from a uniform distribution $g(\phi)$ supported in $[-\delta, \delta]$. If $K$ is large, the law of large numbers yields $r=1 / 2 \delta \int_{-\delta}^{\delta} e^{i \phi} d \phi=$ $\sin \delta / \delta$.

[24] This constant may depend on how we choose the initial conditions. There are two ways to choose initial conditions (ICs). (i) The ICs for the coupling $\lambda+\Delta \lambda$ are the final states when coupling equals to $\lambda$ as suggested in [2], which yields $1 / B=0.6989$. (ii) The ICs are independent, randomly chosen from the interval $[-\pi, \pi]$ for each coupling $\lambda$, resulting in $1 / B=2 / \pi$.

[25] R. Albert and A. Barabasi, Rev. Mod. Phys. 74, 47 (2002).

[26] We observe that the unbiased network generation model of [13] actually generates more hublike networks than the Barabási-Albert algorithm, and hence, we are considering a relatively difficult test case.

[27] T. F. Móri, Comb. Probab. Comput. 14, 339 (2005). 